\title{
Rab7-mediated autophagy regulates phenotypic transformation and behavior of smooth muscle cells via the Ras/Raf/MEK/ERK signaling pathway in human aortic dissection
}

\author{
KESHUAI HE* , HAOLIANG SUN*, JUNJIE ZHANG, RUI ZHENG, JIAXI GU, MING LUO and YONGFENG SHAO
}

\author{
Department of Cardiovascular Surgery, The First Affiliated Hospital of \\ Nanjing Medical University, Nanjing, Jiangsu 210000, P.R. China
}

Received March 8, 2018; Accepted November 19, 2018

DOI: $10.3892 / \mathrm{mmr} .2019 .9955$

\begin{abstract}
Autophagy regulates the metabolism, survival and function of numerous types of cell, including cells that comprise the cardiovascular system. The dysfunction of autophagy has been demonstrated in atherosclerosis, restenotic lesions and hypertensive vessels. As a member of the Ras GTPase superfamily, Rab7 serves a significant role in the regulation of autophagy. The present study evaluated how Rab7 affects the proliferation and invasion, and phenotypic transformations of aortic dissection (AD) smooth muscle cells (SMCs) via autophagy. Rab7 was overexpressed in AD tissues and the percentage of synthetic human aortic SMCs (HASMCs) was higher in AD tissues compared with NAD tissues. Downregulation of Rab7 decreased cell growth, reduced the number of invasive cells and decreased the percentage cells in the G1 phase. Autophagy of HASMCs was inhibited following Rab7 knockdown. Inhibition of autophagy with 3-methyladenine or Rab7 knockdown suppressed the phenotypic conversion of contractile to synthetic HASMCs. The action of Rab7 may be mediated by inhibiting the Ras/Raf/mitogen-activated protein kinase (MAPK) kinase (MEK)/extracellular signal related kinase (ERK) signaling pathway. In conclusion, the results revealed that Rab7-mediated autophagy regulated the behavior of SMCs and the phenotypic transformations in AD via activation of the Ras/Raf/MEK/ERK signaling pathway. The findings of the present study may improve understanding of the role Rab7 in the molecular etiology of AD and suggests
\end{abstract}

Correspondence to: Professor Yongfeng Shao, Department of Cardiovascular Surgery, The First Affiliated Hospital of Nanjing Medical University, 300 Guangzhou Road, Nanjing, Jiangsu 210000, P.R. China

E-mail: syfnjmu@163.com

${ }^{*}$ Contributed equally

Key words: Rab7, phenotypic transformation, smooth muscle cells, autophagy, extracellular signal-regulated kinase the application of Rab7 as a novel therapeutic target in the treatment of human AD.

\section{Introduction}

Acute aortic dissection (AAD) is a common and critical clinical disease with a high rate of mortality; however, its pathogenetic mechanism requires further investigation. Recent studies suggested that AAD arises from problems with vascular function. It is well established that vascular smooth muscle cells (VSMCs) are essential regulators of vascular function (1). VSMCs are located in the middle vascular layer of healthy arteries (2-4), where they secrete vasoconstrictor proteins that help regulate blood vessel tension and blood flow (5). VSMCs are the most important component of the vascular middle layer and may contribute to AAD. VSMCs are divided into two cell phenotypes: Contractile and synthetic $(6,7)$. Fusiform contractile VSMCs demonstrate difficulty in secreting extracellular matrix proteins, and exhibit poor proliferation and migration (8). The proliferative and migration abilities of synthetic VSMCs are enhanced compared with the systolic VSMCs (9-11). Synthetic VSMCs secrete a variety of extracellular matrix components, including collagen, elastin and proteoglycans $(12,13)$. Upon atherosclerosis and arterial restenosis, VSMCs undergo a transformation from the contractile to the synthetic phenotype (14). This transformation promotes the migration of SMCs into the intima, enhances proliferation and promotes the secretion of extracellular proteins (15). These phenotypic transformations may underlie the basis of regulating the composition and stability of blood vessels, which may eventually lead to the formation of vascular lesions (16). Recent studies revealed that extracellular factors and downstream signaling pathways participate in transformation of VSMCs $(17,18)$, such as autophagy.

It was demonstrated that $\mathrm{Rab} 7$ participates in the regulation of VSMC proliferation and migration (2). Autophagy, induced by platelet-derived growth factor, serves an important role in the process of transforming the phenotype of VSMCs from a contractile to a synthetic form by preventing oxidative stress-induced cell death (19). Rab proteins are Ras-related small GTPases, which regulate exo- and endocytic membrane trafficking by vesicle docking and fusion (20). As an important 
member of the Rab GTPase superfamily, Rab7 promotes lysosomal biosynthesis and maintains lysosomal function $(21,22)$. Furthermore, Rab7 serves a pivotal role in the fusion of vesicles and lysosomes, and exhibit an important effect on autophagosome maturation (23). Abnormal expression or alterations in the activity of Rab7 may be associated with cardiovascular diseases, lipid storage disorders and neurodegenerative diseases (24-27). Therefore, the present study hypothesized that phenotypic transformation regulated by Rab7-mediated autophagy may be associated with VSMC proliferation and invasion.

In the present study, AD VSMCs were treated with small interfering (si)RNA or Rab7 overexpression plasmid to assess phenotypic transformations and cellular behavior, including proliferation, invasion, cell cycle and autophagy. The present study aimed to identify the effects of Rab7 on autophagy in VSMCs and determine whether -Rab7-regulated autophagy results in the alteration of VSMC phenotype and cell behavior.

\section{Materials and methods}

Tissue sampling. A total of 51 AAD tissues were obtained from patients with type A AD, who underwent aortic replacement operations at The First Affiliated Hospital of Nanjing Medical University (Nanjing, China) between October 2015 and October 2017 (Ethics no. 2016-SR-144). The main clinicopathological characteristics of the patients are shown in Table I. All experimental protocols were approved by the Ethics Committee of The First Affiliated Hospital of Nanjing Medical University and all patients provided written informed consent. Patients with hereditary connective tissue defects, including Marfan syndrome or traumatic aneurysms, were excluded. The control group comprised 14 non-aortic dissection (NAD) aortic specimens collected from organ donors, containing residual tissues from organ pruning, such as the aortic tissue trimmed during heart transplantations. No significant differences between the groups were observed with respect to the clinical characteristics, including patient age, sex, smoking status, hypertension or diabetes.

Immunohistochemistry. The fresh tissues were fixed in $4 \%$ paraformaldehyde at room temperature for $48 \mathrm{~h}$ dehydrated in a graded ethanol series and embedded in paraffin. Then, $5 \mu \mathrm{m}$ thick sections were dewaxed twice in xylene for $10 \mathrm{~min}$ and rehydrated in ethanol (75\% ethanol $2 \mathrm{~h}, 95 \%$ ethanol $1.5 \mathrm{~h}$, $95 \%$ ethanol $1.0 \mathrm{~h}$, absolute ethyl alcohol $1.5 \mathrm{~h}$ and absolute ethyl alcohol $1.0 \mathrm{~h}$ ) at room temperature. The sections were washed with PBS ( $\mathrm{pH} 7.4) 3$ times for $3 \mathrm{~min}$. Then, the slides were boiled in $10 \mathrm{mM} / 1$ citrate buffer ( $\mathrm{pH}$ 6.0) in an autoclave for $3 \mathrm{~min}$ for antigen retrieval. Following slow cooling, the sections were blocked by immersion in $3 \%$ methanolic peroxide for $15 \mathrm{~min}$ at room temperature and incubated with the following primary antibodies: Antibodies against Rab7 (ab50533; 1:500; Abcam, Cambridge, UK) overnight at $4^{\circ} \mathrm{C}$, followed by horseradish peroxidase (HRP)-conjugated secondary antibodies for $2 \mathrm{~h}$ at room temperature (31430; 1:5,000; Thermo Fisher Scientific, Inc., Waltham, MA, USA). After washing with water, the slides were counterstained with hematoxylin for $2 \mathrm{~min}$ at room temperature, dehydrated and mounted in resin mountant.
Cell culture. Primary human aortic VSMCs (HASMCs) were isolated from the collected tissues using the tissue adhesion method (28); subsequent experiments were performed with 2nd-4th generation cells. Cells were cultured in smooth muscle cell medium (Sciencell Research Laboratories, Inc., San Diego, CA, USA) containing $10 \%$ fetal bovine serum (Sciencell Research Laboratories, Inc.). Cells were grown in cell culture flasks (Corning, Inc., Corning, NY, USA) in a humidified environment in $37^{\circ} \mathrm{C}$ with $5 \% \mathrm{CO}_{2}$.

Cell transfection. siRab7, and Rab7 overexpression and control vectors were obtained from Shanghai GeneChem Co., Inc. (Shanghai, China). Sequences were: siRab7\#1: 5'GUC UAGUUCCCUUCUGUGU(dTdT)3'; siRab7\#2: 5'ACACAG AAGGGAACUAGAC(dTdT)3'. A total of $20 \mathrm{nM}$ of siRNAs were transfected into the cells using RNAiMAX (Invitrogen; Thermo Fisher Scientific, Inc.) according to the manufacturer's protocol. All siRNAs were purchased from Shanghai GeneChem Co., Inc. (Shanghai, China). Transfection efficiency was determined using western blot analysis.

5'Ethynyl-2'-deoxyuridine (EdU) assay. Briefly, the cells were plated into 12-well plates at a density of $8 \times 10^{3}$ cells/well with $500 \mu \mathrm{l}$ culture medium. Cells were fixed at room temperature for 20 min using $4 \%$ paraformaldehyde (PFA), then permeabilized with $0.2 \%$ Triton $\mathrm{X}-100$ at room temperature for $20 \mathrm{~min}$ and blocked with $10 \%$ goat serum (Abcam) in TBS containing $0.05 \%$ Tween-20 (TBST) for $1 \mathrm{~h}$ at room temperature. The EdU assay was performed using the Click-iT Plus EdU Alexa Fluor ${ }^{\circledR}$ 488 Imaging kit (Invitrogen; Thermo Fisher Scientific, Inc.) according to the manufacturer's protocols to determine cell proliferation. Nuclei were counterstained with DAPI at room temperature for $10 \mathrm{~min}$. Under a confocal microscope (Zeiss AG, Oberkochen, Germany), 15 fields/sample (magnification, $\mathrm{x} 200$ ) were observed and the number of EdU-positive nuclei was determined.

Transwell invasion assay. Transwell invasion assays were performed using Transwell chambers (pores, $8 \mathrm{~mm}$; Corning, Inc.). Serum-free medium containing $1 \times 10^{4}$ cells was added to the upper chamber pre-coated with Matrigel (BD Biosciences, San Jose, CA, USA). Following $24 \mathrm{~h}$ incubation in $37^{\circ} \mathrm{C}$ with $5 \% \mathrm{CO}_{2}$ cells that migrated to the lower surface of the membrane were fixed with absolute ethanol and stained with $0.1 \%$ crystal violet at room temperature for $10 \mathrm{~min}$. Cells in the upper chamber were wiped from the Matrigel with a cotton swab. Then, 3 visual fields were randomly selected to screen the cells located on the lower surface of the membrane under an inverted microscope. After calculating the number of cells in every field, the average was counted and analyzed.

Cell cycle analysis. Flow cytometry was performed to detect the cell cycle. Cells were cultured to $80-90 \%$ confluence in 6-well plates. Cells were transfected for $48 \mathrm{~h}$, digested with $0.25 \%$ trypsin at room temperature for $10 \mathrm{~min}$, collected with pre-chilled $70 \%$ ethanol at $4{ }^{\circ} \mathrm{C}$ and maintained at $4{ }^{\circ} \mathrm{C}$ for overnight fixation. Cells were collected by centrifugation at $150 \mathrm{x} \mathrm{g}$ for $10 \mathrm{~min}$ at $4^{\circ} \mathrm{C}$; the upper layer was discarded and ethanol was washed away with PBS. The cells were then stained with PI DNA staining solution (Hangzhou Lianke 
Table I. Association between Rab7 protein expression and clinicopathological characteristics in patients with AD.

\begin{tabular}{lccccc}
\hline Group & $\begin{array}{c}\text { Sex } \\
\text { (female/male) }\end{array}$ & $\begin{array}{c}\text { Age } \\
\text { (years) }\end{array}$ & $\begin{array}{c}\text { Blood } \\
\text { glucose (mmol/l) }\end{array}$ & $\begin{array}{c}\text { Systolic } \\
\text { pressure (mmHg) }\end{array}$ & $\begin{array}{c}\text { Diastolic } \\
\text { pressure (mmHg) }\end{array}$ \\
\hline AD & $7 / 44$ & $47.82 \pm 6.77$ & $4.91 \pm 0.48$ & $155.12 \pm 16.20$ & $84.67 \pm 11.57$ \\
NAD & $2 / 12$ & $45.50 \pm 5.56$ & $4.75 \pm 0.56$ & $125.71 \pm 7.53^{\mathrm{b}}$ & $76.29 \pm 5.64^{\mathrm{c}}$ \\
Relative Rab7 expression & /a & $/$ & $/$ & 0.256 & 0.231 \\
\hline
\end{tabular}

${ }^{\mathrm{a}}$ Not applicable. ${ }^{\mathrm{b}} \mathrm{P}=0.000,{ }^{\mathrm{c}} \mathrm{P}=0.011 . \mathrm{AD}$, aortic dissection; NAD, non-aortic dissection.

Biology Technology, Co., Ltd., Hangzhou, China) at room temperature for 30 min. A Beckman Gallios (AU39633; Beckman Coulter, Inc., Brea, CA, USA) and Wincycle version 3.0 (Beckman Coulter, Inc.) were used for cell cycle analysis.

Detection of autophagy. Immunofluorescence and western blot analysis were used to determine autophagy. For immunofluorescence assays, HASMCs were cultured to $80-90 \%$ confluence in 6-well plates.

The cells were fixed in 4\% PFA at room temperature for $20 \mathrm{~min}$, followed by $0.2 \%$ Triton X-100 for $20 \mathrm{~min}$ and permeabilization with $10 \%$ goat serum in TBST for $1 \mathrm{~h}$ at room temperature. Cells were incubated with rabbit polyclonal anti-microtubule-associated protein light chain 3 (LC3) antibody (ab48394; 1:100; Abcam, Cambridge, UK) at $4^{\circ} \mathrm{C}$ overnight. Cells were then incubated with Alexa Fluor 488-labeled secondary antibody $(5 \mu \mathrm{g} / \mathrm{ml}$; A-11034; Thermo Fisher Scientific, Inc.) for $1 \mathrm{~h}$ at room temperature and imaged using an inverted fluorescence microscope. The expression of Beclin-1 (ab62557; 1:1,000; Abcam), LC3 (ab48394; 1:1,000; Abcam) and P62 (ab56416; 1:1,000; Abcam) were detected by western blotting (described below). Following the administration of 3-methyladenine (3-MA; $5 \mathrm{mM}$ ), a pharmacological inhibitor of autophagy, to Rab7-overexpressing cells at $37^{\circ} \mathrm{C}$ with $5 \% \mathrm{CO}_{2}$ for $48 \mathrm{~h}, \alpha$-SMA (ab5694; 1:1,000; Abcam) and Osteopintin (ab8448; 1:1,000; Abcam) were detected by western blotting (as described below).

Protein extraction and western blotting. Following washes with PBS for 3 times, proteins were extracted from the cells using radioimmunoprecipitation assay lysis buffer containing phenylmethylsulfonyl fluoride and protease inhibitors (R0010; Beijing Solarbio Science \& Technology Co., Ltd., Beijing, China). A Bicinchoninic Acid Protein Assay kit (Beyotime Institute of Biotechnology, Shanghai, China) was used to quantify the total protein. SDS-PAGE (12\%) was used to separate the proteins $(30 \mu \mathrm{g} / \mathrm{lane})$ prior to transfer to polyvinylidene difluoride membranes $(0.45 \mu \mathrm{m}$; EMD Millipore, Billerica, MA, USA). Subsequently, membranes were blocked with TBST and 5\% fat-free dry milk for $2 \mathrm{~h}$ at room temperature. Membranes were incubated with primary antibodies (Beclin-1; ab62557; 1:1,000, LC3; ab48394; 1:1,000 and P62; 1:1,000; ab56416; all from Abcam) in TBST containing 1\% bovine serum albumin (ScienCell Research Laboratories, Inc., San Diego, CA, USA) overnight at $4^{\circ} \mathrm{C}$, followed by incubation with horseradish peroxidase-conjugated secondary antibody at an appropriate dilution with TBST (1:3,000; ab97080 and ab97040; Abcam, UK) at room temperature for $2 \mathrm{~h}$. Membranes were washed three times for $10 \mathrm{~min}$ washes with TBST. A ChemiDoc XRS (Bio-Rad Laboratories, Inc., Hercules, CA, USA) and Supersignal West Femto Trial kit (Thermo Fisher Scientific, Inc.) were used to visualize the bands. Relative protein levels were determined using Image J version $1.45 \mathrm{~s}$ software (National Institutes of Health, Bethesda, MD, USA).

Statistical analysis. All data are presented as the mean \pm standard deviation. SPSS version 17.0 software (SPSS, Inc., Chicago, IL, USA) was used to perform statistical analysis. A Student's t-test was applied to analyze the expression of Rab7 in AD tissues compared with paired NAD tissues. The association between the clinicopathological characteristics of patients and Rab7 expression was determined using Fisher's exact test, analysis of variance (ANOVA) and a Student's t-test. Data were analyzed via one-way ANOVA for 3-group comparisons and two tail student's t-tests for 2 -group comparisons. $\mathrm{P}<0.05$ was considered to indicate a statistically significant difference.

\section{Results}

Rab7 is overexpressed in AD tissue and the abundance of synthetic HASMCs is increased in AD. Rab7 protein expression levels in 14 NAD and 51 AD specimens were determined by western blotting, and were normalized to GAPDH. The results revealed that the expression levels of Rab7 were significantly increased in AD specimens compared with the NAD samples $(\mathrm{P}<0.001$; Fig. 1A). Table I demonstrated the association between Rab7 protein expression and the clinicopathological characteristics in patients with $\mathrm{AD}$. In addition, the expression of Rab7, $\alpha$-SMA and osteopontin was determined by western blotting. It was observed that Rab7 and osteopontin expression was notably increased in the AD specimens, while $\alpha$-SMA expression was decreased compared with the NAD specimens (Fig. 1B). Using immunohistochemistry, it was observed that the AD specimens had significantly higher Rab7 expression compared with in NAD specimens (Fig. 1C and D). These results indicated that the abundance of contractile HASMCs was decreased while synthetic HASMCs were increased in AD $(29,30)$.

Rab7 regulates HASMC proliferation, invasion and cell cycle. As presented in Fig. 1B, Rab7 expression in Case 4 of the AD group was markedly increased compared with the NAD group and Rab7 expression in Case 8 was markedly decreased compared with other AD cases. Thus, Rab7 knockdown was 
A

\section{क}
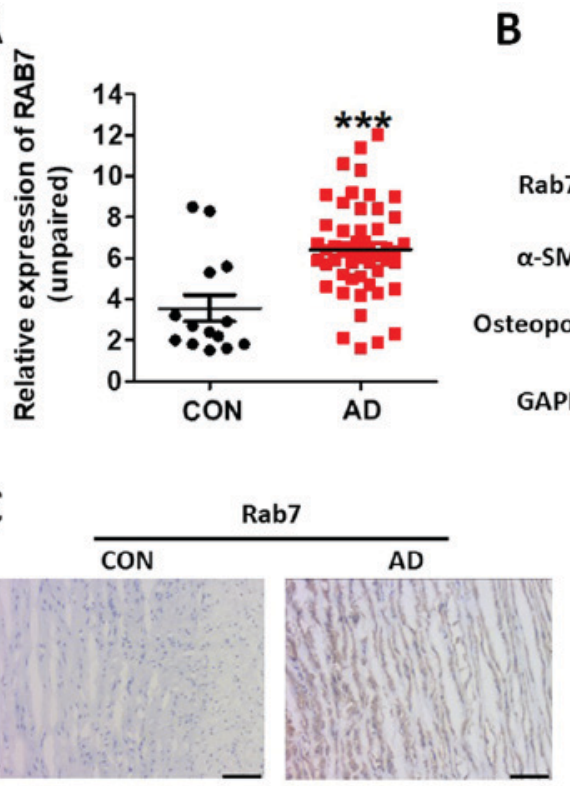

B

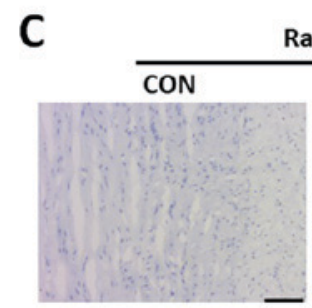

Rab7

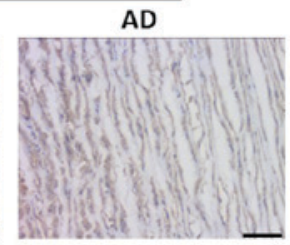

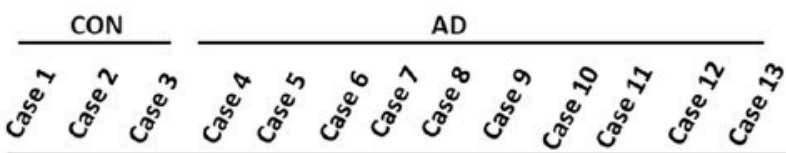

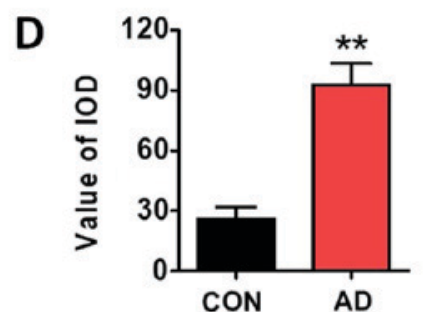

Figure 1. Rab7 expression is increased in AD tissues. (A) Western blotting revealed the upregulation of Rab7 protein in 51 AD tissues compared with 14 NAD tissues. ${ }^{* * *} \mathrm{P}<0.001$ vs. CON. (B) Rab7 and osteopontin protein expression levels increased in 10 AD tissues compared with 3 NAD tissues. $\alpha$-SMA expression levels were decreased in AD tissues compared with the control. (C and D) Representative immunohistochemical staining of Rab7 was stronger in AD samples compared with in NAD tissues. Scale bar, $100 \mu \mathrm{m} .{ }^{* *} \mathrm{P}<0.01$ vs. CON. $\alpha$-SMA, $\alpha$-smooth muscle actin; AD, aortic dissection; CON, control; NAD, non-aortic dissection.

performed in Case 4 HASMCs and Rab7 overexpression was conducted in Case 8 HASMCs to investigate the effects of Rab7 on proliferation, invasion and the cell cycle. The transfection efficiency was demonstrated for the Case 4 knockdown and Case 8 overexpression using western blot analysis (Fig. 2A).

EdU assays were performed to evaluate the effects of Rab7 on proliferation. HASMC proliferation was significantly decreased in Rab7 knockdown compared with the transfected siCtrl Case 4 control (Fig. 2B) and Rab7 overexpression significantly promoted HASMC proliferation compared with the transfected siCtrl Case 8 control (Fig. 2B). Rab7 knockdown in HASMCs using siRab7\#1 and siRab7\#2 was observed to significantly decrease the number of invaded cells compared with the Case 4 control; this was significantly reversed in the Case 8 overexpression group compared with the control (Fig. 2C). The results indicated that Rab7 significantly affected the proliferation and invasion of HASMCs.

Analysis of the cell cycle distribution revealed that Rab7 was able to regulate the HASMC cycle. Compared with the Case 4 control, significantly fewer cells were observed in the G1 phase, and a significantly higher proportion of cells in the $\mathrm{G} 2$ and $\mathrm{S}$ phase were detected following knockdown of Rab7 with siRab7\#1 or siRab7\#2 (Fig. 2D and F). Cell cycle analysis of HASMCs overexpressing Rab7 exhibited opposing results, with a significant increase in G1 cell number, and notable decreases in the $\mathrm{G} 2$ and $\mathrm{S}$ phase cell number compared with the control group (Fig. 2E and G). The results suggested that Rab7 upregulation may induce cell cycle arrest in the G1 phase.

Rab7 regulates autophagy. Immunofluorescence assays were performed to determine the effects of Rab7 on autophagy. Based on the literature, Beclin1, LC3-II and P62 were selected as markers in the evaluation of autophagy.
Immunofluorescence assays demonstrated that HASMC autophagy was significantly decreased following Rab7 knockdown in Case 4 compared with the control cells (Fig. 3A); overexpression promoted HASMC autophagy compared with Case 8 control cells (Fig. 3A). Rab7 knockdown in Case 4 notably decreased Beclin1 and LC3-II, and increased P62 expression compared with the control. These effects were reversed in Rab7 overexpressing cells compared with the Case 8 control (Fig. 3B). This suggested that Rab7 inducted HASMC autophagy.

Rab7 regulates the phenotypic transformations of HASMCs by inducing autophagy. The effects of Rab7 on phenotypic transformations were determined by detecting $\alpha$-SMA and osteopontin expression. $\alpha$-SMA is mainly expressed in contractile HASMCs, while osteopontin is mainly expressed in synthetic HASMCs $(31,32)$. It was demonstrated that $\alpha$-SMA expression was significantly increased in Rab7 knockdown cells of Case 4 compared with the control cells (Fig. 4A) and Rab7 overexpression in Case 8 cells inhibited $\alpha$-SMA expression compared with the control cells (Fig. 4B). Furthermore, osteopontin expression was decreased in Rab7 knockdown cells and increased in Rab7 overexpression cells compared with the Case 4 and Case 8 controls, respectively (Fig. 4A and B). The results indicated that Rab7 significantly promoted HASMC phenotypic transformations from a contractile to a synthetic phenotype.

To further investigate whether the phenotypic transformation of SMCs is regulated by Rab7-mediated autophagy, a pharmacological inhibitor of autophagy, 3-MA, was administered to Rab7-overexpressing cells to suppress autophagy. When autophagy was inhibited by 3-MA, it was observed that $\alpha$-SMA, osteopontin and LC3 expression was markedly altered compared with the control (Fig. 4B). In conclusion, Rab7 was 
A
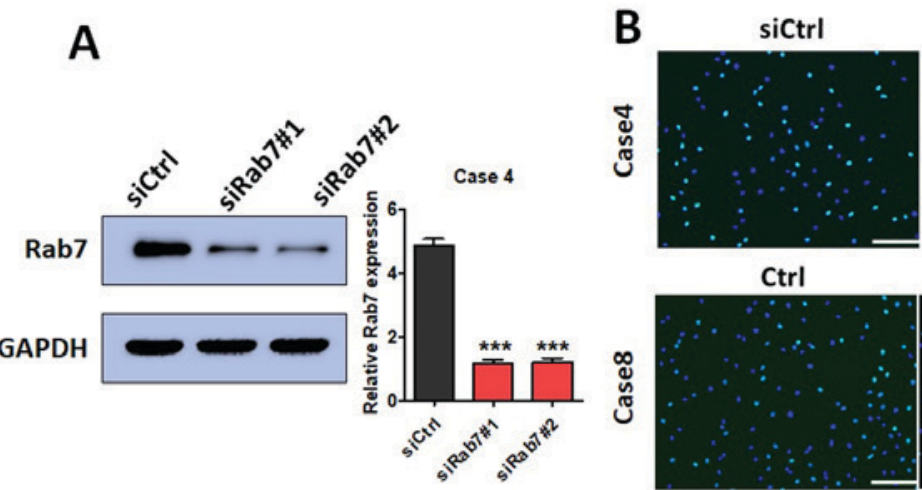

C
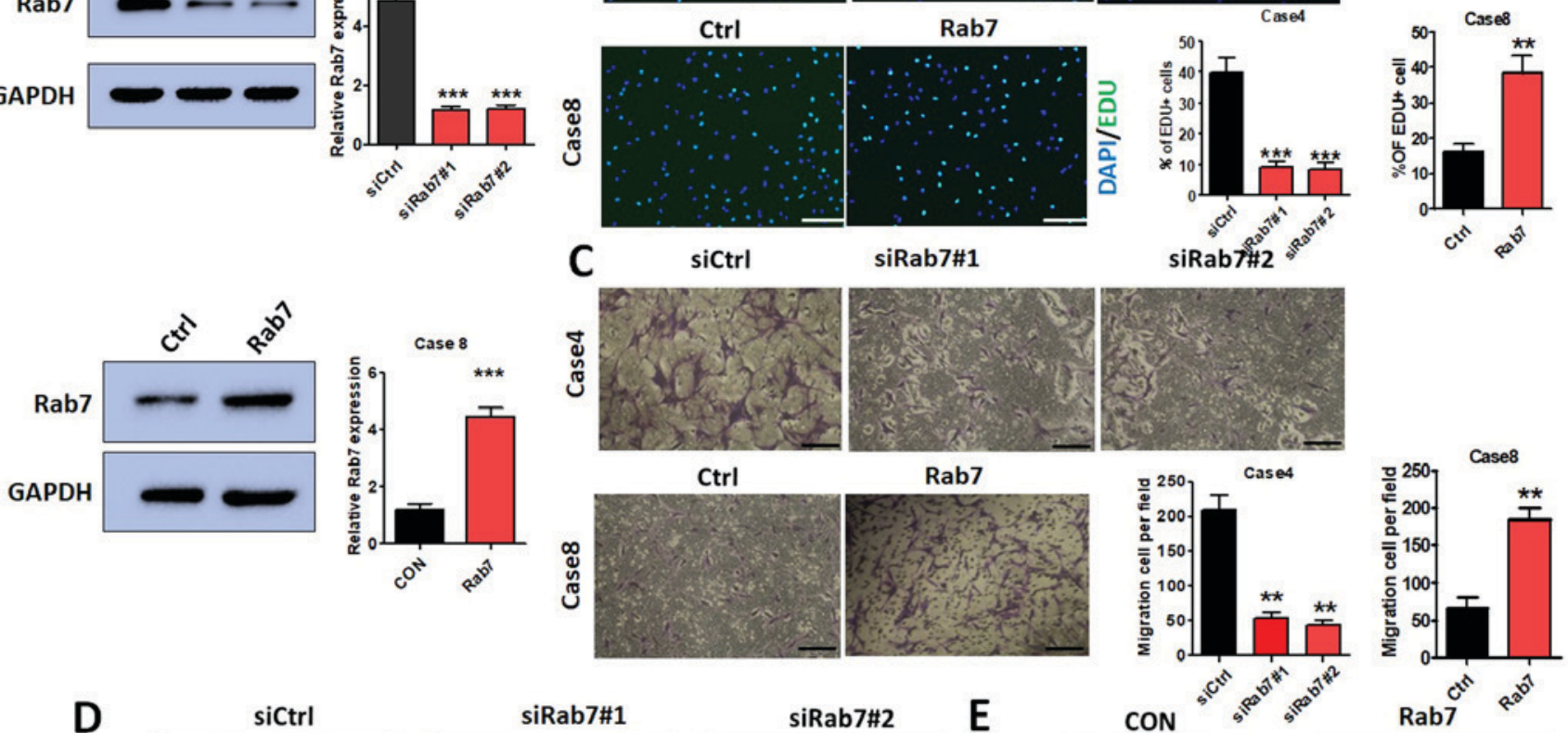

D

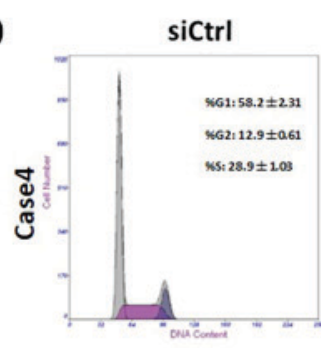

$\mathbf{F}$

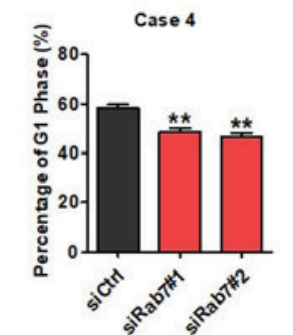

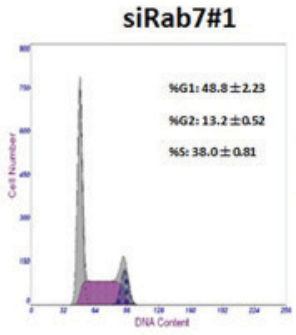

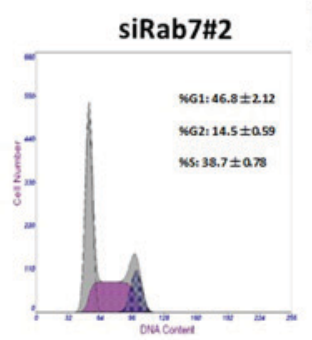

E
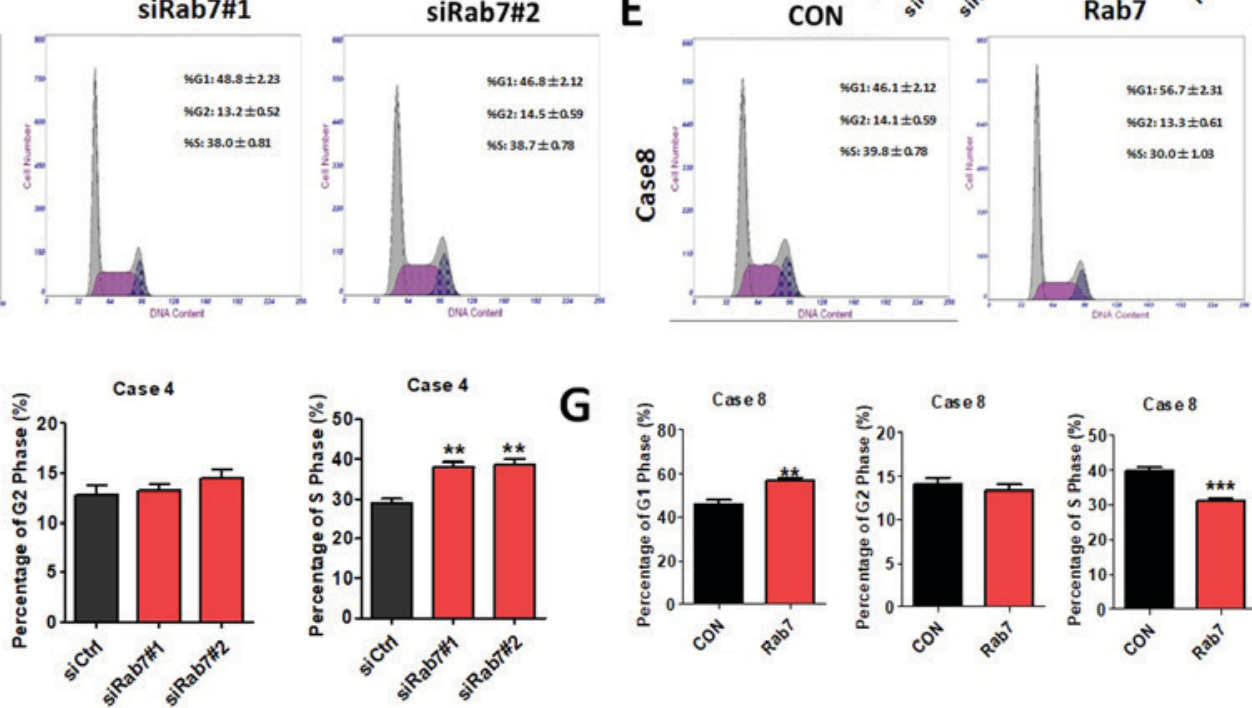

G

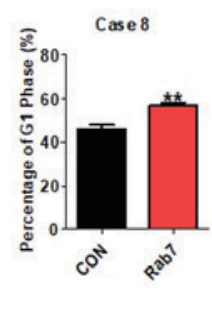

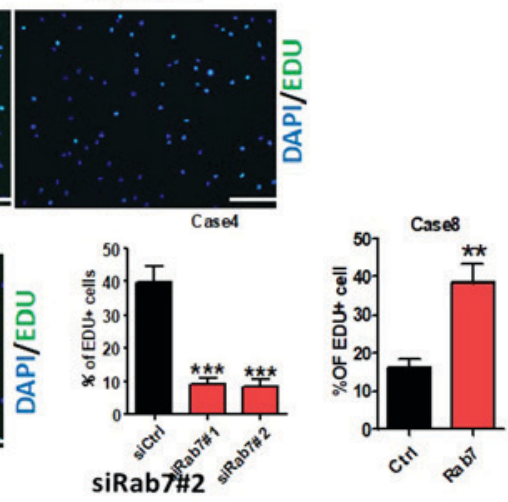

siRab7\#2

Figure 2. HASMC proliferation and invasion is regulated by Rab7 in vitro. (A) Transfection efficiency was demonstrated for the Case 4 knockdown and Case 8 overexpression using western blot analysis. (B) Rab7 knockdown inhibited Case 4 HASMC proliferation and overexpression promoted Case 8 HASMC proliferation as determined by an EdU assay. ${ }^{* *} \mathrm{P}<0.01$ and ${ }^{* * *} \mathrm{P}<0.001$ vs. CON. Scale bar $=200 \mu \mathrm{m}$. (C) In vitro, HASMC invasion decreased in the Rab7 knockdown group and increased in the Rab7 overexpression group compared with the respective controls. ${ }^{* *} \mathrm{P}<0.01 \mathrm{vs}$. CON. Scale bar $=200 \mu \mathrm{m}$. (D and F) HASMC cell cycle was regulated by Rab7 in vitro. Rab7 knockdown induced G1 arrest in Case 4 HASMCs compared with the control (** $\mathrm{P}<0.01$ vs. CON); the proportion of cells in the $\mathrm{S}$ phase was increased ( ${ }^{* *} \mathrm{P}<0.01$ vs. $\left.\mathrm{CON}\right)$ and the proportion of cells in $\mathrm{G} 2$ phase markedly changed ( $\mathrm{P}>0.05 \mathrm{vs}$. $\left.\mathrm{CON}\right)$. (E and $\left.\mathrm{G}\right) \mathrm{Rab} 7 \mathrm{overexpres-}$ sion in Case 8 HASMCs increased the number of cells in $\mathrm{G} 1$ phase $\left({ }^{* *} \mathrm{P}<0.01 \mathrm{vs}\right.$. CON $)$; the number of cells in $\mathrm{S}$ phase decreased $\left({ }^{* * * *} \mathrm{P}<0.001\right.$ vs. CON) and that of $\mathrm{G} 2$ cells markedly changed (P>0.05 vs. CON). CON, control; EdU, 5'ethynyl-2'-deoxyuridine; HASMCs, human aortic smooth muscle cells; siCtrl, small interfering RNA control; siRab7, siRNA Rab7.

observed to induce autophagy, which may be associated with the phenotypic transformation of HASMCs.

Rab7 activates the Ras/Raf/mitogen-activated protein kinase (MAPK) kinase (MEK)/extracellular signal-regulated kinase (ERK) signaling pathway in HASMCs. The Ras/Raf/MEK/ERK signaling pathway serves an important role in autophagy $(33,34)$. The expression levels of various molecules of the Ras/Raf/MEK/ERK signaling pathway were analyzed by western blotting. Rab7 knockdown resulted in decreased expression of K-Ras, phosphorylated (p)-BRAF, p-MEK and p-ERK in Case 4 cells compared with the transformed siCtrl control (Fig. 5A). In Rab7-overexpressing cells, the expression of K-Ras, p-BRAF, p-MEK and p-ERK was increased in Case 8 cells compared with the control (Fig. 5B). As the Ras/Raf/MEK/ERK signaling pathway 
A

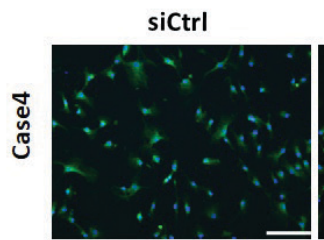

Ctrl
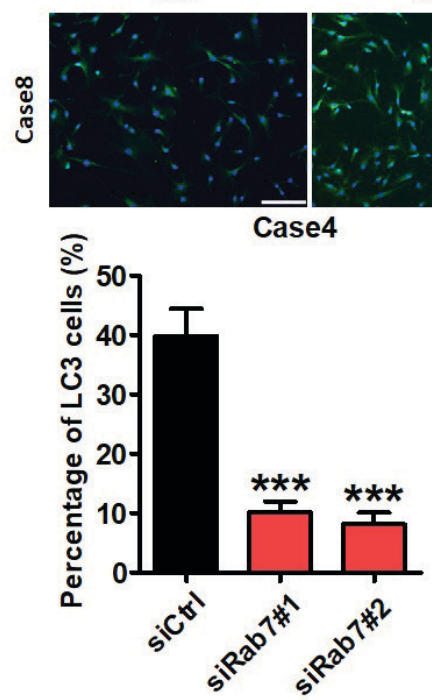

siRab7\#1

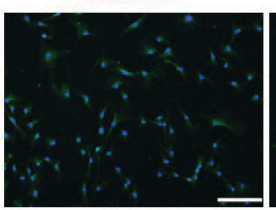

Rab7
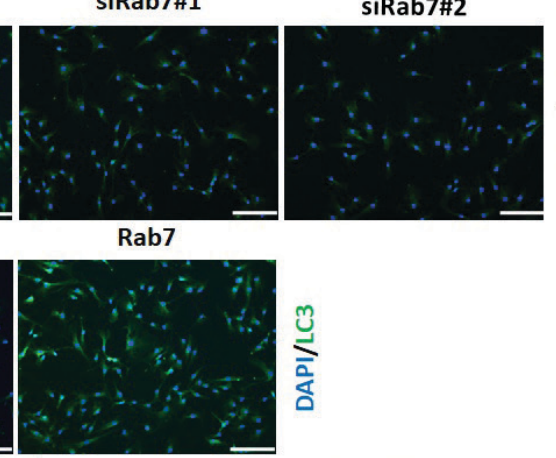

B
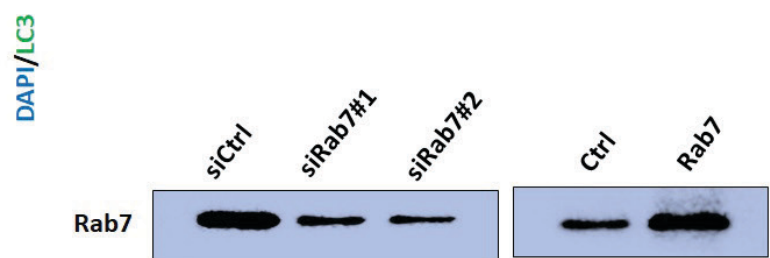

Beclin1
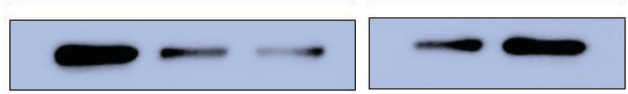

LC-3 I
LC-3 II
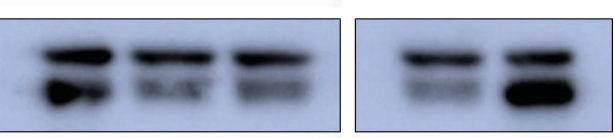

P62

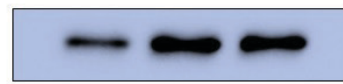

GAPDH
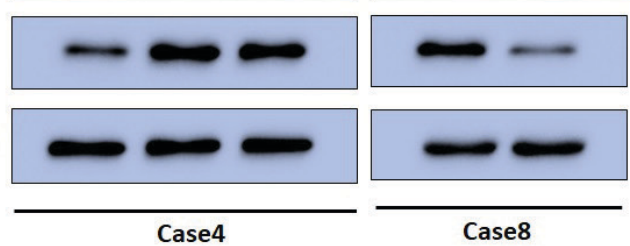

Figure 3. HASMC autophagy is regulated by Rab7 in vitro. (A) Immunofluorescence assays revealed that LC3 expression was decreased in Rab7 knockdown cells compared with the control (Case $4 ;{ }^{* * *} \mathrm{P}<0.001$ vs. Ctrl) and upregulated in Rab7 overexpressing HASMCs compared with the control $\left(\mathrm{Case} 8 ;{ }^{* *} \mathrm{P}<0.01\right.$ vs. Ctrl). Scale bar $=200 \mu \mathrm{m}$. (B) Rab7, Beclin1 and LC3-II protein levels decreased in siRab\#1 and siRab\#2 groups, and P62 protein levels were increased compared with the siCtrl group (Case 4) as determined by western blotting. Overexpression of Rab7 notably increased the expression of Rab7, Beclin1 and LC3-II, and downregulated that of P62 levels compared with the control (Case 8). Scale bar=200 $\mu \mathrm{m}$. Ctrl, control; HASMCs, human aortic smooth muscle cells; LC3, microtubule-associated protein light chain 3; siCtrl, small interfering RNA control; siRab7, siRNA Rab7.

is associated with autophagy (35), the results of the present study suggested that Rab7 induced autophagy by activating the Ras/Raf/MEK/ERK signaling pathway in HASMCs.

\section{Discussion}

Analyses into the pathogenesis of AD have become more widespread and various factors that contribute to the development of AD have been identified, such as hereditary diseases, including Marfan syndrome, hypertension, atherosclerosis and autoimmune diseases (36-38); however, the molecular mechanism underlying the development and progression of $\mathrm{AD}$ remains unknown. It is well established that normal aortic media comprises numerous regularly arranged VSMCs and extracellular matrix (39). As SMCs are associated with the structure and function of the aorta, there is the potential for serving important roles in AD development (40-42). In healthy individuals, contractile VSMCs serve a crucial role in maintaining vascular wall structure and function (43). In patients with AD, a significant increase in the number and ratio of synthetic VSMCs was observed, which resulted in decreased aortic elasticity and rupture of the vessel wall $(44,45)$. These studies suggested that the phenotypic conversion of VSMCs may be the key factor underlying the occurrence and development of AD.

Previous studies have reported that numerous factors, including vasoconstrictor agonists and extracellular matrices regulate the phenotypic conversion of VSMCs $(46,47)$. While contractile VSMCs express $\alpha$-SMA extensively, synthetic VSMCs exhibit high expression of osteopontin (31). The present study reported that Rab7 downregulation resulted in increased $\alpha$-SMA and osteopontin expression. These results suggested that $\mathrm{Rab} 7$ may promote the phenotypic conversion of contractile to synthetic VSMCs; however, the specific mechanism remains unclear. Rab7, a member of the Rab GTPase superfamily, serves an unique role in the regulation of autophagy (48). Several studies have revealed that autophagy promotes the phenotypic conversion of contractile to synthetic VSMCs $(19,49,50)$. Therefore, the present study proposed that autophagy may be affected by the phenotypic transformations VSMCs mediated by Rab7. To further verify this hypothesis, the autophagy inhibitor 3-MA was applied to cells to investigate whether Rab7 promotes the phenotypic transformations of VSMCs. It was observed that the effects of Rab7 on phenotypic transformations were reversed following autophagy inhibition. In conclusion, autophagy may be a key component in Rab7-inducing phenotypic transformations. The effects of Rab7 on phenotypic transformations were revealed via the suppression of autophagy in Rab7 knockdown cells; the phenotypic transformations were associated with autophagy in Rab7 overexpressing cells.

Furthermore, as autophagy is regulated by the Ras/Raf/MEK/ERK signaling pathway $(33,35)$, it was proposed that Rab7 may induce autophagy-mediated phenotypic transformations via the Ras/Raf/MEK/ERK signaling 

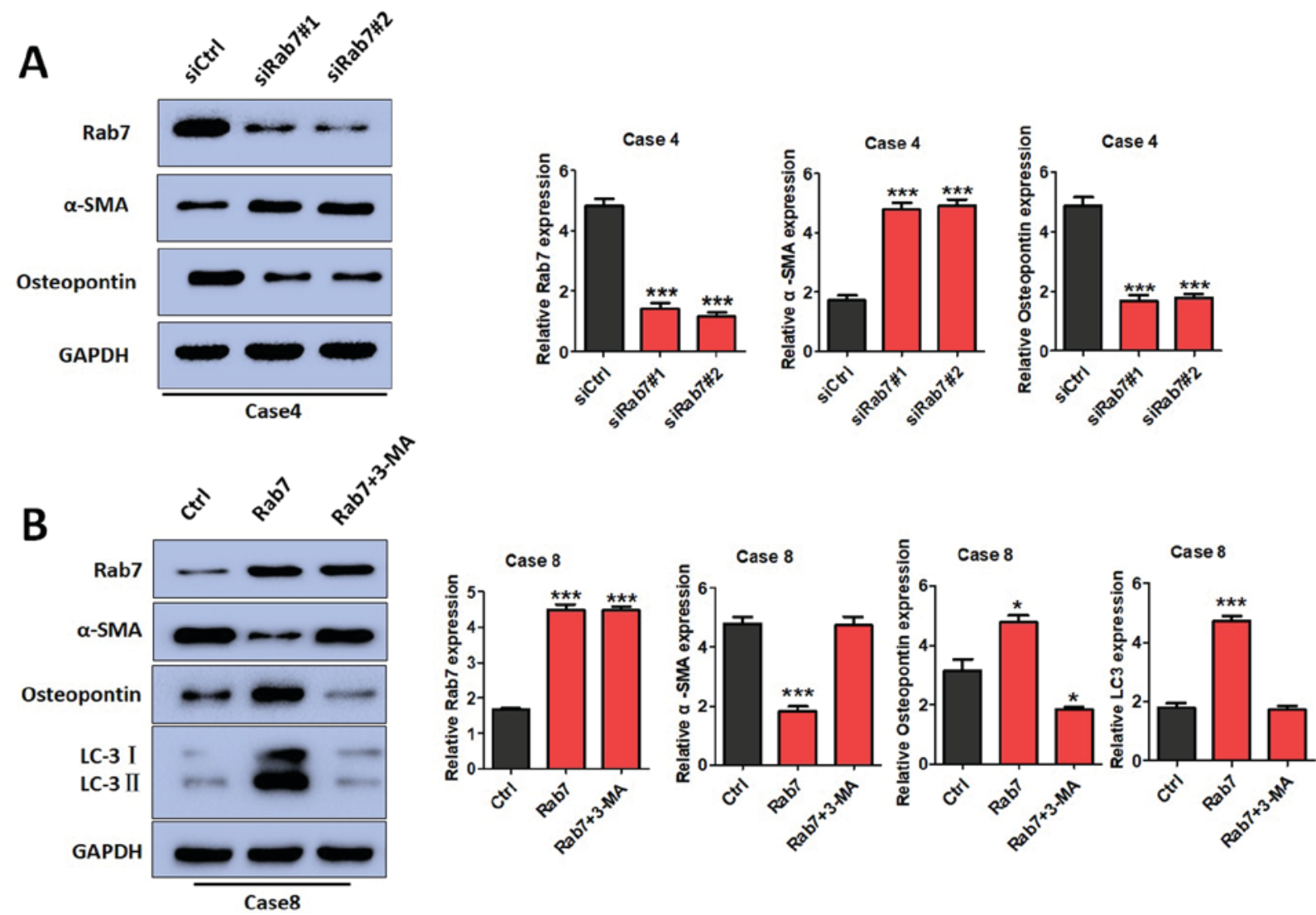

Figure 4. HASMC phenotypic transformations are regulated by Rab7 in vitro. (A) Western blotting revealed Rab7 downregulation in Case 4 HASMCs. Following Rab7 knockdown, $\alpha$-SMA expression was notably increased and osteopontin levels were decreased compared with the control. ${ }^{* * *} \mathrm{P}<0.001 \mathrm{vs}$. CON. (B) Overexpression of Rab7 promoted phenotypic transformation in Case 8 HASMC as indicted by alterations in the expression of $\alpha$-SMA and osteopontin. 3-MA was used to inhibit autophagy; the expression of $\alpha$-SMA and osteopontin was markedly altered in Rab7-overexpressing cells (Case 8). ${ }^{* * * *} \mathrm{P}<0.001,{ }^{*} \mathrm{P}<0.05$ vs. CON. 3-MA, 3-methyladenine; Ctrl, control; HASMC, human aortic smooth muscle cells; LC3, microtubule-associated protein light chain 3; siCtrl, small interfering RNA control; siRab7, siRNA Rab7.
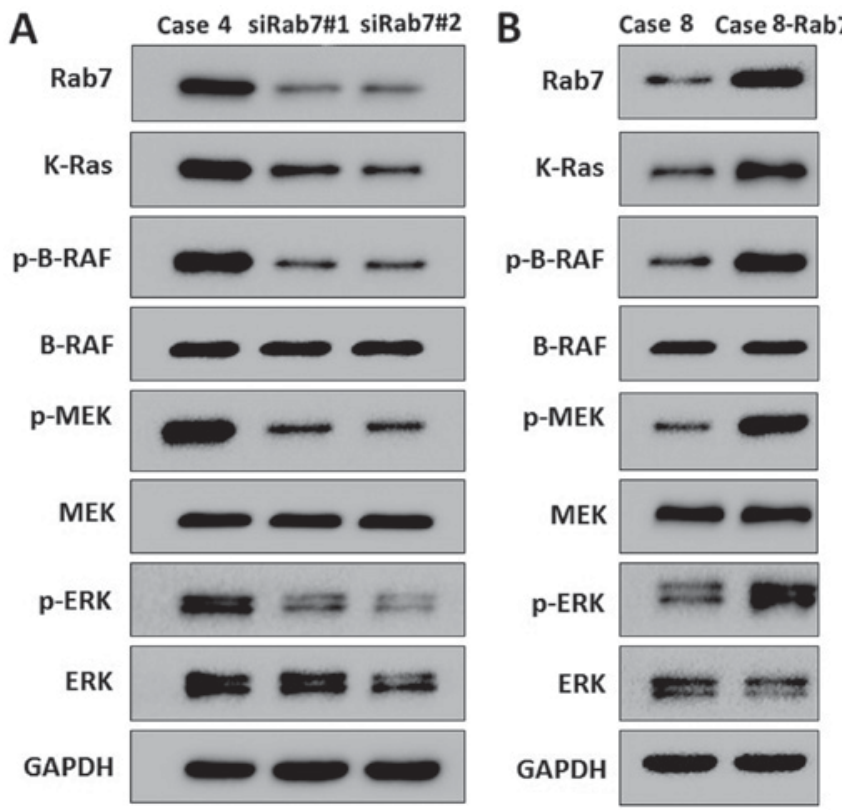

Figure 5. Rab7 promotes the Ras/Raf/MEK/ERK signaling pathway. Expression of proteins associated with the Ras/Raf/MEK/ERK signaling pathway in Rab7 knockdown (A; Case 4) and overexpression (B; Case 8) in human aortic smooth muscle cells were analyzed by western blotting. MAPK, mitogen-activated protein kinase; MEK, MAPK kinase; p, phosphorylated; siRab7, small interfering RNA Rab7. pathway. It was observed that Rab7 knockdown or overexpression regulated the expression of Ras/Raf/MEK/ERK signaling pathway-associated proteins. Future experiments are required investigate the effects of the Ras/Raf/MEK/ERK signaling pathway on Rab7-mediated VSMC phenotypic transformations.

The present study revealed that Rab7 promoted VSMC proliferation and invasion in AD. This phenomenon may be associated with autophagy and phenotypic transformations. Autophagy has been considered to be the basic catabolic mechanism that is essential for cell survival, differentiation, development and the cellular response to stress via the regulating the actions of numerous lysosomes (51-53). In aortic endothelial cells, autophagy promotes tube formation and endothelial cell migration by inducing angiogenesis (54). Additionally, in lung cancer cells, autophagy facilitates proliferation, migration and invasion (55). VSMCs of the synthetic phenotype have enhanced proliferative and migration abilities compared with the contractile phenotype $(56,57)$. Compared with healthy controls, the present study positively correlated VSMC proliferation and invasion with autophagy and phenotypic transformations in AD. In the present study, autophagy and phenotypic transformations were reported to be regulated by Rab7, which in turn was supported by Rab7 promoting VSMC proliferation and invasion in AD; however, the exact 
mechanism of action remains unclear and requires further investigation.

There are certain limitations to the present study. Synthetic VSMCs synthesize and secrete extracellular matrices (58); however, the expression of extracellular matrix proteins, including collagen and elastin were not detected in the present study. Therefore, the role of Rab7 and the Ras/Raf/MEK/ERK signaling pathway in extracellular matrix-associated protein secretion remains unknown. Additionally, as VSMC phenotypic conversion is associated with AD development and various cardiovascular events including coronary heart disease and vascular complications in diabetes (59), further investigation into phenotypic transition is essential for the prevention and treatment of particular cardiovascular diseases.

In summary, Rab7 promoted the proliferation and invasion of VSMCs in AD, which may be mediated by the regulation of autophagy and phenotypic transformation. Additionally, Rab7 expression was associated with the Ras/Raf/MEK/ERK signaling pathway. These results indicate the important role of Rab7 in the etiology of AD and suggested that Rab7 may be considered as a novel therapeutic target for the treatment of $\mathrm{AD}$; however, further experiments are required to determine the underlying mechanism of Rab7 in the regulation of autophagy and phenotypic transformation.

\section{Acknowledgements}

Not applicable.

\section{Funding}

The present study was supported by the ' 333 ' Project of Jiangsu Province (grant no. BRA2014336) and the Natural Science Foundation of Jiangsu Province (grant no. BK20151590).

\section{Availability of data and materials}

All data generated or analyzed during this study are included in this published article.

\section{Authors' contributions}

$\mathrm{KH}$ and HS were in charge of completing most of the experiments. $\mathrm{JZ}, \mathrm{RZ}, \mathrm{JG}$ and ML aided in the collection of specimens. JZ and RZ performed western blotting, and JG and ML conducted the cell experiments. YS provided project design and guidance.

\section{Ethics approval and consent to participate}

The present study was approved by the Ethics Committee of The First Affiliated Hospital of Nanjing Medical University (Nanjing, China) and all patients provided written informed consent.

\section{Patient consent for publication}

Not applicable.

\section{Competing interests}

The authors declare that they have no competing interests.

\section{References}

1. Hecht E, Freise C, Websky KV, Nasser H, Kretzschmar N, Stawowy P, Hocher B and Querfeld U: The matrix metalloproteinases 2 and 9 initiate uraemic vascular calcifications. Nephrol Dial Transplant 31: 789-797, 2016.

2. Liu K, Fang C, Shen Y, Liu Z, Zhang M, Ma B and Pang X: Hypoxia-inducible factor 1a induces phenotype switch of human aortic vascular smooth muscle cell through PI3K/AKT/AEG-1 signaling. Oncotarget 8: 33343-33352, 2017.

3. Zhong Y, Feng J, Li J and Fan Z: Curcumin prevents lipopolysaccharide-induced matrix metalloproteinase-2 activity via the Ras/MEK1/2 signaling pathway in rat vascular smooth muscle cells. Mol Med Rep 16: 4315-4319, 2017.

4. Freise C, Bobb V and Querfeld U: Collagen XIV and a related recombinant fragment protect human vascular smooth muscle cells from calcium-/phosphate-induced osteochondrocytic transdifferentiation. Exp Cell Res 358: 242-252, 2017.

5. Owens GK, Kumar MS and Wamhoff BR: Molecular regulation of vascular smooth muscle cell differentiation in development and disease. Physiol Rev 84: 767-801, 2004.

6. Hogarth DK, Sandbo N, Taurin S, Kolenko V, Miano JM and Dulin NO: Dual role of PKA in phenotypic modulation of vascular smooth muscle cells by extracellular ATP. Am J Physiol Cell Physiol 287: C449-C456, 2004.

7. Pei C, Qin S, Wang M and Zhang S: Regulatory mechanism of human vascular smooth muscle cell phenotypic transformation induced by NELIN. Mol Med Rep 12: 7310-7316, 2015.

8. Yan Y, Wang C, Lu Y, Gong H, Wu Z, Ma X, Li H, Wang B and Zhang X: Mineralocorticoid receptor antagonism protects the aorta from vascular smooth muscle cell proliferation and collagen deposition in a rat model of adrenal aldosterone-producing adenoma. J Physiol Biochem 74: 17-24, 2018.

9. Lv P, Zhang F, Yin YJ, Wang YC, Gao M, Xie XL, Zhao LL, Dong LH, Lin YL, Shu YN, et al: SM22 $\alpha$ inhibits lamellipodium formation and migration via Ras-Arp2/3 signaling in synthetic VSMCs. Am J Physiol Cell Physiol 311: C758-C767, 2016.

10. Potier M, Gonzalez JC, Motiani RK, Abdullaev IF, Bisaillon JM, Singer HA and Trebak M: Evidence for STIM1- and Orail-dependent store-operated calcium influx through ICRAC in vascular smooth muscle cells: Role in proliferation and migration. FASEB J 23: 2425-2437, 2009.

11. Guo RW, Yang LX, Li MQ, Pan XH, Liu B and Deng YL: Stim1and Orail-mediated store-operated calcium entry is critical for angiotensin II-induced vascular smooth muscle cell proliferation. Cardiovasc Res 93: 360-370, 2012.

12. Meng YH, Tian C, Liu L, Wang L and Chang Q: Elevated expression of connective tissue growth factor, osteopontin and increased collagen content in human ascending thoracic aortic aneurysms. Vascular 22: 20-27, 2014.

13. Wanjare M, Kusuma S and Gerecht S: Defining differences among perivascular cells derived from human pluripotent stem cells. Stem Cell Reports 2: 561-575, 2014.

14. Pahk K, Joung C, Jung SM, Young Song H, Yong Park J, Woo Byun J, Lee YS, Chul Paeng J, Kim C, Kim S and Kim WK: Visualization of synthetic vascular smooth muscle cells in atherosclerotic carotid rat arteries by F-18 FDG PET 7: 6989, 2017.

15. Mack CP: Signaling mechanisms that regulate smooth muscle cell differentiation. Arterioscler Thromb Vasc Biol 31: 1495-1505, 2011.

16. Pyle AL and Young PP: Atheromas feel the pressure: Biomechanical stress and atherosclerosis. Am J Pathol 177: 4-9, 2010.

17. Ma J, Feng Y, Li Z and Tang C: The effect of adrenomedullin and proadrenomedullin $\mathrm{N}$-terminal 20 peptide on angiotensin II induced vascular smooth muscle cell proliferation. Iran J Basic Med Sci 19: 49-54, 2016.

18. Chuang TD, Pearce WJ and Khorram O: miR-29c induction contributes to downregulation of vascular extracellular matrix proteins by glucocorticoids. Am J Physiol Cell Physiol 309: C117-C125, 2015.

19. Salabei JK, Cummins TD, Singh M, Jones SP, Bhatnagar A and Hill BG PDGF-mediated autophagy regulates vascular smooth muscle cell phenotype and resistance to oxidative stress. Biochem J 451: 375-388, 2013

20. Mima J: Reconstitution of membrane tethering mediated by Rab-family small GTPases. Biophys Rev 10: 543-549, 2018. 
21. Distefano MB, Kjos I, Bakke O and Progida C: Rab7b at the intersection of intracellular trafficking and cell migration. Commun Integr Biol 8: e1023492, 2015.

22. Jimenez-Orgaz A, Kvainickas A, Nägele H, Denner J, Eimer S Dengjel $\mathrm{J}$ and Steinberg F: Control of RAB7 activity and localization through the retromer-TBC1D5 complex enables RAB7-dependent mitophagy. EMBO J 37: 235-254, 2018.

23. Noda T and Yoshimori T: Between canonical and antibacterial autophagy: Rab7 is required for GAS-containing autophagosome-like vacuole formation. Autophagy 6: 419-420, 2010.

24. Cataldo AM, Mathews PM, Boiteau AB, Hassinger LC Peterhoff CM, Jiang Y, Mullaney K, Neve RL, Gruenberg J and Nixon RA: Down syndrome fibroblast model of Alzheimer-related endosome pathology: Accelerated endocytosis promotes late endocytic defects. Am J Pathol 173: 370-384, 2008.

25. Zhang M, Chen L, Wang S and Wang T: Rab7: Roles in membrane trafficking and disease. Biosci Rep 29: 193-209, 2009

26. Ismail S, Gherardi MJ, Froese A, Zanoun M, Gigoux V, Clerc P, Gaits-Iacovoni F, Steyaert J, Nikolaev VO and Fourmy D Internalized receptor for glucose-dependent insulinotropic peptide stimulates adenylyl cyclase on early endosomes. Biochem Pharmacol 120: 33-45, 2016.

27. Han J, Pan XY, Xu Y, Xiao Y, An Y, Tie L, Pan Y and Li XJ: Curcumin induces autophagy to protect vascular endothelial cell survival from oxidative stress damage. Autophagy 8: 812-825, 2012

28. Corbera-Bellalta M, Planas-Rigol E, Lozano E, TerradesGarcía N, Alba MA, Prieto-González S, García-Martínez A Albero R, Enjuanes A, Espígol-Frigolé G, et al: Blocking interferon $\gamma$ reduces expression of chemokines CXCL9, CXCL10 and CXCL11 and decreases macrophage infiltration in ex vivo cultured arteries from patients with giant cell arteritis. Ann Rheum Dis 75: 1177-1186, 2016.

29. Sun HJ, Ren XS, Xiong XQ, Chen YZ, Zhao MX, Wang JJ, Zhou YB, Han Y, Chen Q, Li YH, et al: NLRP3 inflammasome activation contributes to VSMC phenotypic transformation and proliferation in hypertension. Cell Death Dis 8: e3074, 2017.

30. Zhang X, Zhao JF, Zhao F, Yan JF, Yang F, Huang XJ, Chen G, $\mathrm{Fu} \mathrm{HY}$ and $\mathrm{Lv} \mathrm{BD}$ : The protective effect of salidroside on hypoxia-induced corpus cavernosum smooth muscle cell phenotypic transformation. Evid Based Complement Alternat Med 2017: $3530281,2017$.

31. Cao C, Ji X, Luo X and Zhong L: Gingipains from Porphyromonas gingivalis promote the transformation and proliferation of vascular smooth muscle cell phenotypes. Int J Clin Exp Med 8: 18327-18334, 2015.

32. Tian L, Chen K, Cao J, Han Z, Gao L, Wang Y, Fan Y and Wang C: Galectin-3-induced oxidized low-density lipoprotein promotes the phenotypic transformation of vascular smooth muscle cells. Mol Med Rep 12: 4995-5002, 2015

33. Butler DE, Marlein C, Walker HF, Frame FM, Mann VM, Simms MS, Davies BR, Collins AT and Maitland NJ: Inhibition of the PI3K/AKT/mTOR pathway activates autophagy and compensatory Ras/Raf/MEK/ERK signalling in prostate cancer. Oncotarget 8: 56698-56713, 2017.

34. Wang Y, Nie H, Zhao X, Qin Y and Gong X: Bicyclol induces cell cycle arrest and autophagy in HepG2 human hepatocellular carcinoma cells through the PI3K/AKT and Ras/Raf/MEK/ERK pathways. BMC Cancer 16: 742, 2016.

35. Xin L, Ma X, Xiao Z, Yao H and Liu Z: Coxsackievirus B3 induces autophagy in HeLa cells via the AMPK/MEK/ERK and Ras/Raf/MEK/ERK signaling pathways. Infect Genet Evol 36: 46-54, 2015

36. Agg B, Benke K, Szilveszter B, Pólos M, Daroczi L, Odler B, Nagy ZB, Tarr F, Merkely B and Szabolcs Z: Possible extracardiac predictors of aortic dissection in Marfan syndrome. BMC Cardiovasc Disord 14: 47, 2014

37. Gaddum NR, Keehn L, Guilcher A, Gomez A, Brett S, Beerbaum P, Schaeffter T and Chowienczyk P: Altered dependence of aortic pulse wave velocity on transmural pressure in hypertension revealing structural change in the aortic wall. Hypertension 65: 362-369, 2015.

38. Nienaber CA, Clough RE, Sakalihasan N, Suzuki T, Gibbs R, Mussa F, Jenkins MP, Thompson MM, Evangelista A, Yeh JS, et al: Aortic dissection. Nat Rev Dis Primers 2: 16053, 2016.

39. Lacolley P, Regnault V, Segers P and Laurent S: Vascular smooth muscle cells and arterial stiffening: Relevance in development, aging, and disease. Physiol Rev 97: 1555-1617, 2017.

40. Docherty CK, Carswell A, Friel E and Mercer JR: Impaired mitochondrial respiration in human carotid plaque atherosclerosis: A potential role for Pink1 in vascular smooth muscle cell energetics. Atherosclerosis 268: 1-11, 2018
41. Iida Y, Tanaka H, Sano H, Suzuki Y, Shimizu H and Urano T: Ectopic expression of PCSK9 by smooth muscle cells contributes to aortic dissection. Ann Vasc Surg 48: 195-203, 2018.

42. Wei X, Sun Y, Wu Y, Zhu J, Gao B, Yan H, Zhao Z, Zhou J and Jing Z: Downregulation of Talin-1 expression associates with increased proliferation and migration of vascular smooth muscle cells in aortic dissection. BMC Cardiovasc Disord 17: 162, 2017.

43. Yang L, Gao L, Nickel T, Yang J, Zhou J, Gilbertsen A, Geng Z, Johnson C, Young B, Henke C, et al: Lactate promotes synthetic phenotype in vascular smooth muscle cells. Circ Res 121: 1251-1262, 2017.

44. Perrucci GL, Rurali E, Gowran A, Pini A, Antona C, Chiesa R, Pompilio G and Nigro P: Vascular smooth muscle cells in Marfan syndrome aneurysm: The broken bricks in the aortic wall. Cell Mol Life Sci 74: 267-277, 2017.

45. Cai YL and Wang ZW: The expression and significance of IL-6, IFN- $\gamma$, SM22 $\alpha$, and MMP-2 in rat model of aortic dissection. Eur Rev Med Pharmacol Sci 21: 560-568, 2017.

46. Zhou W, Liu W, Liao H, Cao Z, Xie H, Zhang S and Chen M: Testosterone suppresses oxidized low-density lipoprotein-induced vascular smooth muscle cell phenotypic transition and proliferation. Xi Bao Yu Fen Zi Mian Yi Xue Za Zhi 31: 775-778, 2015 (In Chinese).

47. Wang L, Zheng J, Du Y, Huang Y, Li J, Liu B, Liu CJ, Zhu Y, Gao Y, $\mathrm{Xu} \mathrm{Q}$, et al: Cartilage oligomeric matrix protein maintains the contractile phenotype of vascular smooth muscle cells by interacting with alpha(7)beta(1) integrin. Circ Re 106: 514-525, 2010.

48. Zhan L, Chen S, Li K, Liang D, Zhu X, Liu L, Lu Z, Sun W and $\mathrm{Xu}$ E: Autophagosome maturation mediated by Rab7 contributes to neuroprotection of hypoxic preconditioning against global cerebral ischemia in rats. Cell Death Dis 8: e2949, 2017.

49. Song TF, Huang LW, Yuan Y, Wang HQ, He HP, Ma WJ, Huo LH, Zhou $\mathrm{H}$, Wang $\mathrm{N}$ and Zhang TC: LncRNA MALAT1 regulates smooth muscle cell phenotype switch via activation of autophagy. Oncotarget 9: 4411-4426, 2017

50. Zhao Y, Guo Y, Jiang Y, Zhu X, Liu Y and Zhang X: Mitophagy regulates macrophage phenotype in diabetic nephropathy rats. Biochem Biophys Res Commun 494: 42-50, 2017.

51. Levine B and Klionsky DJ: Development by self-digestion: Molecular mechanisms and biological functions of autophagy. Dev Cell 6: 463-477, 2004.

52. Matsuo T and Sadzuka Y: Extracellular acidification by lactic acid suppresses glucose deprivation-induced cell death and autophagy in B16 melanoma cells. Biochem Biophys Res Commun 496: 1357-1361, 2018.

53. Grootaert MOJ, Moulis M, Roth L, Martinet W, Vindis C, Bennett MR and De Meyer GRY: Vascular smooth muscle cell death, autophagy and senescence in atherosclerosis. Cardiovasc Res 114: 622-634, 2018.

54. Slevin M, Krupinski J, Rovira N, Turu M, Luque A, Baldellou M, Sanfeliu C, de Vera N and Badimon L: Identification of pro-angiogenic markers in blood vessels from stroked-affected brain tissue using laser-capture microdissection. BMC Genomics 10: 113, 2009.

55. Zhan Z, Xie X, Cao H, Zhou X, Zhang XD, Fan H and Liu Z: Autophagy facilitates TLR4- and TLR3-triggered migration and invasion of lung cancer cells through the promotion of TRAF6 ubiquitination. Autophagy 10: 257-268, 2014.

56. Stone JD, Narine A, Shaver PR, Fox JC, Vuncannon JR and Tulis DA: AMP-activated protein kinase inhibits vascular smooth muscle cell proliferation and migration and vascular remodeling following injury. Am J Physiol Heart Circ Physiol 304: H369-H381, 2013.

57. Xu F, Ahmed AS, Kang X, Hu G, Liu F, Zhang W and Zhou J: MicroRNA-15b/16 attenuates vascular neointima formation by promoting the contractile phenotype of vascular smooth muscle through targeting YAP. Arterioscler Thromb Vasc Biol 35: 2145-2152, 2015.

58. Yan C: Cyclic nucleotide phosphodiesterase 1 and vascular aging. Clin Sci (Lond) 129: 1077-1081, 2015.

59. McCarty MF and DiNicolantonio JJ: The molecular biology and pathophysiology of vascular calcification. Postgrad Med 126: 54-64, 2014.

This work is licensed under a Creative Commons Attribution-NonCommercial-NoDerivatives 4.0 International (CC BY-NC-ND 4.0) License. 